\title{
Effect of spin glass order on magnetic polarons in semimagnetic semiconductors
}

\author{
A.L. Chudnovskiy, R. Oppermann, B. Rosenow \\ Inst. f. Theoret. Physik, Universität Würzburg, D-970\%4 Würzburg, F.R.Germany \\ D.R. Yakovlev *, U. Zehnder, W. Ossau \\ Physikalisches Institut, Universität Würzburg, D-97074 Würzburg, F.R.Germany
}

(November 5, 2018)

\begin{abstract}
A theory accounting for the specific features of magnetic polarons (MP) in the presence of spin glass order is presented. We derive and solve selfconsistent equations for i) the polaron magnetisation, ii) the thermodynamically averaged carrier-spin, and iii) for the spin glass order parameter. The temperature dependence of these quantities is analysed in detail. The modification of the spin glass phase due to the presence of the exchange field of the carrier inside the magnetic polaron volume is investigated. The onset of spin glass order leads to a plateau-like flattening in the temperature dependence of the MP energy at low temperatures. It is found that solutions of spin glass equations are needed to optimally fit the experimental data of the temperature dependence of the exciton magnetic polaron (EMP) energy in (Cd,Mn)Te. Moreover, the dynamical aspects of the MP formation are discussed. Our model predicts qualitatively different temperature dependences of the MP formation time in different dynamical scenarios.
\end{abstract}

\section{INTRODUCTION}

Diluted magnetic semiconductors (DMS) exhibit a variety of magnetooptical effects (giant Zeeman splitting, giant Faraday rotation etc.) which allow for the inysstigation of these materials with both standard magnetometric and optical techniques (for detailed review see Ref.4). The DMS (Cd,Mn)Te has been studied intensively during the last decades $2-17$, its magnetic properties are determined by the spin $5 / 2$ of the $\mathrm{Mn}^{2+}$-ions substituting Cd-ions randomly on the geometrically frustrated fcc lattice. Excitons are prominent quasiparticles in this material and probe locally the magnetic state of the system. Under suitable conditions they align the surroundig manganese spins and turn into polarons. The properties of these new excitations are determined by a variety of sample parameters like quality and structure of barrier surfaces. However, in all probes with sufficiently high manganese content (or at temperatures below $1 \mathrm{~K}$ ) the disorder induced spin glass $(\mathrm{SG})$ phase must be taken into account. We focus on this aspect of polaronic DMS physics.

The magnetically active manganese ions are coupled via an antiferromagnetic interaction whose strength falls off rapidly with distance (the next-nearest neighbor (NNN) coupling is one order smaller than the nearest-neighbor $(\mathrm{NN})$ interaction, which equals $-5.7 \mathrm{~K}$ ). By increasing the manganese concentration, clusters of magnetically coupled $\mathrm{Mn}$-ions are formed. At concentrations above $20 \%$ a percolating Mn-cluster emerges, exhibiting a transition into a spin glass phase at low (helium) temperatures, which is caused by the combination of geometric frustration and site randomness 8 . 10 . The SG phase is characterised by the order parameter $q=\lim _{t \rightarrow \infty}\langle S(0) S(t)\rangle$, where $t$ is the observation time, $S(0)$ and $S(t)$ denote spins at identical sites and at times 0 and $t$, and brackets represent the thermodynamic average. In zero field $q$ is zero in the paramagnetic phase and assumes a nonzero value in the spin glass phase 19 .

Experimentally the SG phase transition has been observed by a cusp in the temperature dependence of the magnetic susceptibility and by the onset of irreversipility in the field cooled and zero field cooled measurements of the remanent magnetisation (for reviews see Refs.6.18). 19). The explicit use of spin glass equations for fitting experimental data of the magnetic polaron energy is however new and a key point of the present work.

The manganese ions interact strongly with electronic states in the valence and conduction bands. In magnetic fields this leads to the giant Zeeman splitting of the band states, which extends up to tenths of meV. In the absence of an external magnetic field the formation of the magnetic polaron (MP) state - a carrier with a cloud of magnetically polarised Mn spins which has a lowered energy - becomes possible. The dominant contribution to this energy gain comes from the exchange interaction of manganese spins with the hole-spin. Magaetic polarons were investigated in detail for the case of carriers bound to a shallow impurity (for review see Ref.6) and for excitons localised by compositional fluctuations. 14 .

Since the properties of the magnetic polaron are strongly influenced by the magnetic state of the system, one can expect that the formation of the spin glass phase should affect the properties of this quasiparticle. The onset of SG order should show up for example in the temperature dependence of the MP energy. The aim of the present work is to analyse theoretically the effects related to the interaction between the magnetic polaron and SG order, proving 
that SG effects are specific, hence distinguishable from any kind of antiferromagnetic clustering effects, and indeed required to explain qualitatively and quantitatively the new experimental data.

The paper is organized as follows. In section [II a model which accounts for the interaction between spin glass and magnetic polaron is presented and selfconsistent equations are derived for this model. An analytical analysis of these equations is presented in section III followed by detailed numerical evaluations and a comparison of the theoretical results with the experimental data in section IV. Finally in section $\mathrm{V}$ an extended model, which takes into account dynamical aspects of magnetic polaron formation in the presence of spin glass order is presented.

\section{THE MODEL AND ITS COUPLED POLARON SPIN GLASS EQUATIONS}

In this paper we consider the magnetic part of the MP energy, which is directly influenced by the SG ordering of the Mn spin system22 23. The exchange energy can also lead to the shrinking of the wave function of the polaron, thus contributing indirectly to the change of the kinetic energy of the MP. This contribution will be neglected throughout this paper. Furthermore, the contribution of the hole dominates the exchange energy of MP in $(\mathrm{Cd}, \mathrm{Mn}) \mathrm{Te}$ 14, 23. For simplicity we account for the hole contribution only, neglecting the small part of the exchange energy gained via coupling between electron and spin glass. These assumptions are surely justified for localised excitons.

We adopt here the Villain model of Ising pseudospinst. In Refl detailed arguments were given in favor of this model and its applicability to (Cd,Mn)Te. The lower critical dimension for its Ising SG transition being smaller than three together with the fact that the Dzyaloshinskii-Moriya interaction, which would also turn the Heisenferg into an Ising SG (provided this coupling is isotropic itself; anisotropy would lead to special universality classese), is too small, supports the above model description. In the model considered here each pseudospin belongs to a tetrahedron with Mn spins on the corners. Due to the nearest neighbor antiferromagnetic interaction between the Mn spins the tetrahedron is frustrated and its ground states can be characterised by a mixed product $\xi_{i j k}=\sigma_{\mathbf{i}} \cdot\left(\sigma_{\mathbf{j}} \times \sigma_{\mathbf{k}}\right)$ of three neighboring spins which can assume only two opposite values. The normalised value of this product $S=\xi /|\xi|$ can be 1 or -1 and is hence considered as an Ising pseudospin.

The interaction between hole- and Mn-spins will be described by the following model. Consider a percolating cluster with $N$ pseudospins in $(\mathrm{Cd}, \mathrm{Mn}) \mathrm{Te}$ with random frustrated interaction between the spins leading to spin glass order at low temperatures. We assume the interaction between Ising pseudospins to be of infinite range. The hole spin of the exciton interacts with a part of $N_{1}=\alpha N$ pseudospins of the entire spin cluster. In this way the finite range of this exchange coupling is taken into account. We represent this Kondo-like coupling by $-\left(\kappa / N_{1}\right) s^{z} \sum_{i=1}^{N_{1}} S_{i}$. Here $\kappa$, $s^{z}$, and $S_{i}$ denote the effective exchange constant, $z$-component of the hole spin, and the Ising pseudospin at site $i$ respectively. The "exchange box"-model is assumed, i.e. the wave function of the hole is constant inside the magnetic polaron and zero outside21. Our assumption implies $|\Psi(r)|^{2}=1 / N_{1}$ inside the polaron and zero outside. Therefore the Hamiltonian of the model is given by

$$
H=-\frac{\kappa}{N_{1}} s^{z} \sum_{i=1}^{N_{1}} S_{i}-\sum_{(i, j)}^{N} J_{i j} S_{i} S_{j}
$$

The term $-\sum_{(i, j)}^{N} J_{i j} S_{i} S_{j}$ describes the random interaction between spins of the spin glass with gaussian-distributed random $J_{i j}$ of width $J$ and the convention that $(i, j)$ counts pairs $i, j$ only once and only with $i \neq j$.

To investigate the physical effects which result from this model we express the partition function employing the replicatrick, perform the configuration average over the frustrated spin couplings, and after the decoupling procedure by use of the Hubbard-Stratonovich transformation obtain the saddle point equations for the model (for the detailed description of this technique see Ref 18 ). The partition function of the model is thus obtained as

$$
\left\langle Z^{n}\right\rangle=\prod \int P\left(J_{i j}\right) d J_{i j} \sum_{S_{i}, s= \pm 1} \exp \left[\sum_{a=1}^{n} \beta\left\{\frac{\kappa}{N_{1}} s^{a} \sum_{i=1}^{N_{1}} S_{i}^{a}+\sum_{i, j}^{N} J_{i j} S_{i}^{a} S_{j}^{a}\right\}\right],
$$

with $\beta \equiv 1 / T . P\left(J_{i j}\right)$ denotes the Gaussian distribution of couplings, the superscripts $a$ relate to replicas. Here and in what follows the superscript $z$ will be dropped for the hole-spin $s$.

The Kondo-term $-\left(\kappa / N_{1}\right) s \sum_{i=1}^{N_{1}} S_{i}$ is decoupled with the help of the identity

$$
s \sum_{i=1}^{N_{1}} S_{i}=\frac{1}{2}\left(\left(s+\sum_{i=1}^{N_{1}} S_{i}\right)^{2}-s^{2}-\left(\sum_{i=1}^{N_{1}} S_{i}\right)^{2}\right)
$$


The spin glass term in the exponent of the partition function is identical to the hamiltonian of the wellknown Sherrington-Kirkpatrick (SK) model (see 180 ). Thus the decoupling of this term is completely equivalent to that of the SK model.

The whole decoupling procedure transforms the partition function into

$$
\begin{aligned}
\left\langle Z^{n}\right\rangle= & \sum_{s, S_{i}} \int \prod d V_{a} d M_{a} d R_{a} d Q^{a b} \exp \left[\beta \sum _ { a = 0 } ^ { n } \left\{-\frac{\kappa}{2}\left(N_{1} R_{a}^{2}+N_{1} M_{a}^{2}+1 / N_{1} V_{a}^{2}\right)\right.\right. \\
& \left.+\kappa\left(R_{a}+i V_{a} / N_{1}\right) s^{a}+\kappa\left(R_{a}+i M_{a}\right) \sum_{i=1}^{N_{1}} S_{i}^{a}\right\} \\
& \left.+\sum_{a, b}^{n}\left\{-\beta^{2} \frac{N J^{2}}{4}\left(Q^{a b}\right)^{2}+\beta^{2} \frac{J^{2}}{2} \sum_{i=1}^{N} Q^{a b} S_{i}^{a} S_{i}^{b}\right\}\right],
\end{aligned}
$$

where the fields $R_{a}, V_{a}, M_{a}$ facilitate the decoupling of the Kondo-like interaction, while the field $Q^{a b}$ does the job for the spin glass interaction.

Now we define the replica-symmetric and spatially homogeneous saddle point Ansatz for the decoupling fields by

$$
\begin{aligned}
R_{a} & =\eta \\
M_{a} & =i m \\
V_{a} & =i p \\
Q^{a b} & =q, \text { for } a \neq b .
\end{aligned}
$$

For the fields $Q^{a b}$, replica-diagonal and replica-offdiagonal components are distinguished in general. The replicaoffdiagonal components are rejałed to the SG order parameter, whereas the replica diagonal components are noncritical for the Ising spin glass model18. After the substitution of the saddle-point Ansatz in the partition function the free energy of the system is obtained as follows

$$
\begin{aligned}
\beta F= & \frac{1}{2} \frac{N_{1}}{N} \beta \kappa\left(\eta^{2}-m^{2}-\left(p / N_{1}\right)^{2}\right)+\frac{\beta^{2} J^{2}}{2}\left[(q-1)^{2}\right. \\
& -\frac{N_{1}}{N} \int_{z}^{G} \ln \cos h[\beta(J \sqrt{q} z+\kappa(\eta-m))]-\frac{N-N_{1}}{N} \int_{z}^{G} \ln \cosh [\beta J \sqrt{q} z],
\end{aligned}
$$

where

$$
\int_{z}^{G} f(z) \equiv \frac{1}{\sqrt{2 \pi}} \int d z e^{-z^{2} / 2} f(z) .
$$

The free energy becomes extremised by $\eta, p, m$ and $q$ satisfying the following saddle point equations

$$
\begin{gathered}
\eta=p / N_{1}+m \\
p=\tanh [\beta \kappa m] \\
m=\int_{z}^{G} \tanh \left[\beta\left(J \sqrt{q} z+\kappa \frac{p}{N_{1}}\right)\right] \\
q=\frac{N_{1}}{N} \int_{z}^{G} \tanh ^{2}\left[\beta\left(J \sqrt{q} z+\kappa \frac{p}{N_{1}}\right)\right] \\
+\frac{N-N_{1}}{N} \int_{z}^{G} \tanh ^{2}[\beta J \sqrt{q} z]
\end{gathered}
$$

The value $q=\frac{N_{1}}{N}\left\langle S^{a} S^{b}\right\rangle_{\text {ins }}+\frac{N-N_{1}}{N}\left\langle S^{a} S^{b}\right\rangle_{o}(a \neq b)$ is the weighted average of Edwards-Anderson order parameters inside and outside the polaron respectively 1 . $p=\left\langle s^{a}\right\rangle$ represents the thermodynamically averaged spin of the hole 
and $m=1 / N_{1}\left\langle\sum_{i=1}^{N_{1}} S_{i}^{a}\right\rangle$ denotes the average magnetisation in the polaron. The equations (12)-(14) describe the whole phase diagram of the model in the static mean field approximation. From the qualitative analysis of these equations three characteristic temperature ranges can be identified.

(i) At high temperatures $T>\kappa / \sqrt{N_{1}}$ the equations have only trivial solutions. The system is paramagnetic and thermal fluctuations suppress the magnetic polaron formation.

(ii) At $\kappa / \sqrt{N_{1}}>T>J$ the magnetic polaron is formed but the Mn system remains in the paramagnetic phase.

(iii) At $T<J$ the SG order is expected to show up in the Mn system. Hence the value $\kappa / \sqrt{N_{1}}$ determines the temperature of the MP formation. In the experiments the MP is formed in the paramagnetic temperature regime, which implies the inequality $\kappa / \sqrt{N_{1}}>J$.

\section{TEMPERATURE DEPENDENCES OF MAGNETISATION IN THE POLARON AND OF THE SPIN GLASS ORDER PARAMETER IN THE VICINITY OF THE PHASE TRANSITION}

Here we examine the dependence of the SG order parameter $q$ and of the cluster magnetisation $m$ on $T-J$, where $J$ corresponds to the transition temperature of an independent SG model. The main difference between the present case described by equation (14) and the analogous equation for the independent spin glass 18 is caused by the presence of the exchange field

$$
h_{\text {exch }}=\kappa \frac{p}{N_{1}}
$$

which the localised hole exerts on the pseudospin. It is worth noting that the influence of the exchange field on the magnetisation in the polaron and on the SG order parameter $q$ is analogous to the influence of an external magnetic field localized in the volume of the magnetic polaron (see Eqs.(13) and (14)). The nonzero magnetic field competes with the exchange interaction between Mn spins and destroys partially their grdering. This results in the smearing of the phase transition and lowering of the (smeared) transition temperature 18 . The transition should still show up in a change of the temperature behavior of the magnetization $m$ and of the SG order parameter $q$. In order to see these expected changes we analyse the temperature behavior of the quantities $m$ and $q$ in the vicinity of the point $T=J$. The case when the polaron constitutes a fixed part of the Mn spin system is considered, i.e. $\alpha=\frac{N_{1}}{N}$ fixed, $0<\alpha<1$. We expand the right-hand sides of Eqs. 13), (14) for small $q$ up to quadratic terms. After integration over the random field $z$ the equation for $q$ becomes

$$
2 \beta^{4} J^{4} q^{2}+\left(1-\beta^{2} J^{2}(1-4 C)\right) q-C=0,
$$

where $C \equiv \alpha \tanh ^{2}\left(\beta h_{\text {exch }}\right)$. The physical solution for $q$ reads

$$
q=\frac{\beta^{2} J^{2}-1-4 \beta^{2} J^{2} C+\sqrt{\left(1-\beta^{2} J^{2}\right)^{2}+8 \beta^{2} J^{2} C}}{4 \beta^{4} J^{4}} .
$$

In the region $\left|1-\beta^{2} J^{2}\right| \gg \beta^{2} J^{2} C$ the behavior of $q$ changes (for $C=0$ nonanalytically) at $\beta J=1$. For $T>T_{c}$ (i.e. in the paramagnetic phase)

$$
q \approx C \approx \frac{\alpha h_{e x c h}^{2}}{T_{c}^{2}}(1-2 \theta),
$$

where $\theta \equiv T / T_{c}-1$. In the SG-phase, $T<T_{c}$,

$$
q \approx 2 \frac{h_{e x c h}^{2}}{T_{c}^{2}}(1+2 \theta)-\theta .
$$

The dependence $q(\theta)$ is linear both above and below $T_{c}$, but one observes different slopes in the paramagnetic phase and in the SG phase. Similar behavior is observed in the magnetisation $m$. We find

$$
m \approx\left\{\begin{array}{cl}
\frac{h_{e x c h}}{T_{c}}(1-\theta) & \text { for } T>T_{c}, \\
\frac{h_{e x c h}}{T_{c}}-\alpha \frac{h_{e x c h}^{3}}{T_{c}^{3}}(1+25 \theta) & \text { for } T<T_{c} .
\end{array}\right.
$$

The linear dependence of $m$ on $\theta$ becomes very weak below $T_{c}$, i.e. in the SG phase.

The solutions of the saddle point equations describe also the smearing of the otherwise sharp phase transition as caused by the finite exchange field in the magnetic polaron. The smearing is efficient around the point $T_{c}=J$ within the temperature interval given by $\left|1-\beta^{2} J^{2}\right| \ll \beta^{2} J^{2} C$. Beyond this interval the power law behavior of $m$ and $q$ characteristic of the transition in the pure spin glass is retrieved. 


\section{NUMERICAL SADDLE-POINT SOLUTIONS AND COMPARISON WITH EXPERIMENTS}

In order to obtain a detailed description of the physical behavior we solved the saddle-point equations (12)-(14) numerically. It is convenient to define a composite SG order parameter according to $q=\alpha q_{1}+(1-\alpha) q_{2}$, where

$$
\begin{gathered}
q_{1} \equiv \int_{G} d z \tanh ^{2}\left(\beta\left(J \sqrt{q} z+\kappa \frac{p}{N_{1}}\right)\right), \\
q_{2} \equiv \int_{G} d z \tanh ^{2}(\beta J \sqrt{q} z) .
\end{gathered}
$$

describe spin glass order inside $\left(q_{1}\right)$ and outside $\left(q_{2}\right)$ the magnetic polaron, respectively.

A qualitative understanding of the coupled selfconsistent solutions involving polarisation $p$, magnetisation $m$, and the SG order parameters $q_{1}, q_{2}$ can be obtained as follows. Fig.1 displays the magnetisation, which is determined from Eq. (13), and the SG order parameters. The thermal behavior of $m$ and $q_{1}$ is strongly affected by the coupling $\kappa_{1} \equiv \kappa / N_{1}$ which controls the interaction energy of a pseudospin with the hole-spin. For a large value $\kappa_{1}=3.14 m e V$ (curve 1 in Fig.1) the magnetisation $m$ decreases monotonuously with temperature and the phase transition appears strongly smeared by a large exchange field (which keeps $q_{1}$ at high values due to non spin glass contributions and thus inhibits its critical decay at the zero field freezing temperature). At smaller $\kappa_{1}=0.628 \mathrm{meV}$ (curve 2 in Fig.1) instead, $m(T)$ displays a maximum near the almost sharp SG transition signalled by the rather steep decay of both SG order parameters $q_{1}$, within the MP, and $q_{2}$. This is in accordance with the analytical solution given in Eq.(20). For fixed temperature, $m$ and $q_{1}$ grow monotonuously with $\kappa_{1}$. The temperature dependence of the average hole-spin $p$ at two different values of the interaction energy $\kappa$ between hole-spin and pseudospins within the MP is shown in Fig.2. The change of $\kappa$ affects the thermal behavior of the polarisation $p(T)$ in a similarly strong way as a $\kappa_{1}-$ variation alters the magnetisation $m(T)$. In turn, the spin glass order parameter $q_{2}$ outside the magnetic polaron depends very weakly both on $\kappa$ and $\kappa_{1}$. Fig.1 shows that with increasing $\kappa_{1}$ the temperature dependence of $q_{1}$, which resembles the $q_{2}$-curve for small $\kappa_{1}$ (almost sharp SG transition), changes over and approaches the magnetisation curve $m(T)$ in the large $\kappa_{1}$-regime.

The total number $N$ of pseudospins enters in all formulae only in the form $N_{1}=\alpha N$. Since, additionally, the distinction between magnetic order inside the MP and the one within the remaining spin glass domain (not exposed to the exchange field) will influence the quantitative agreement between theory and experiment, it is convenient to analyse thermal properties as a function of $N_{1}$ and $\alpha . N_{1}$ together with $\kappa$ determines the exchange energy $\kappa_{1}$ on a pseudospin. Changing $N_{1}$, at fixed $\kappa$ and $\alpha$, is thus equivalent to tuning $\kappa_{1}$, which was defined in the preceding paragraph. The parameter $\alpha$ determines the relative extension of the polaron and enters in the SG order parameter via the relation $q=\alpha q_{1}+(1-\alpha) q_{2}$. Large values of $\alpha$ correspond to a strong influence of the polaronic exchange field on the spin glass order because $\alpha$ is the weight of the $q_{1}$-contribution to the composite SG order parameter $q$. As $\alpha$ decreases, the peak $m(T)$ shifts to higher temperatures and becomes more pronounced (see Fig.3).

Measurements of the magnetic-field-induced circular polarisation degree of exciton luminescence (reflecting the magnetic susceptibility 12 ) and of the magnetic polaron energy (determined by selective excitation of localised excitons 14 ) against temperature are displayed in Fig. 1 . The polarisation degree shows a cusp at $T=8 \mathrm{~K}$ characteristic of the spin glass phase transition 11.6 . The cusp temperature coincides with the SG transition temperature, which was obtained for this manganese concentration by other measurements (see Ref. 10 ). The experiments on the temperature dependent EMP energy (solid circles in Fig. (1) do not show any peculiarity at the spin glass transition temperature $T_{c}=8 \mathrm{~K}$. However, a plateau is observed at lower temperatures. We argue that this plateau results from the onset of SG order inside the polaron. The plateau extends over a temperature range lying below the cusp temperature of the zero field spin glass, because of the large exchange field inside the MP.

In order to determine the parameters of our model we fitted the experimentally known temperature dependence of the exciton magnetic polaron (EMP) energy in a $\mathrm{Cd}_{0.67} \mathrm{Mn}_{0.33}$ Te epilayer. The exchange part of this energy is calculated according to

$$
\epsilon=\kappa m p
$$

We fix the value of $J=8 K$ (the cusp temperature in the circular polarisation data) whereas the effective exchange interaction $\kappa$ between a hole spin and pseudospins (tetrahedrae of Mn spins) in the polaron is chosen as a fit parameter. We also vary the parameters $N$ and $\alpha$ to obtain maximal agreement of the calculation with the experimental points. The best fit, given by the solid line in Fig. A, is obtained with $\alpha=1, N=20, \kappa=40.5 \mathrm{meV}$, which is already in a rather good agreement with the experimental data. This justifies our assumption that the magnetic contribution 
to the EMP energy dominates thermal variations and is hence responsible for the main features of its temperature dependence. The numerical calculations indicate that for these parameters the hole spin is completely polarised, which means $p=1$ for all temperatures under consideration. Thus the temperature dependence of the EMP-energy reflects the polarisation degree of the $\mathrm{Mn}$ spins in the region of the excitonic wave function. Calculating the exchange field $(\sim \kappa /(\alpha N))$ in the polaron we obtain a value of about 4.7T, which is in reasonable agreement with the value $5.9 \mathrm{~T}$ obtained by other experimental techniques 12 . If we take into account that $N$ is the number of pseudospins representing tetrahedrae of $\mathrm{Mn}$ spins, we can as well estimate the number of $\mathrm{Mn}$ spipsinside the polaron to be about 60 , which is roughly a factor of 2 smaller than the value estimated from experiment 6 . 2 .

Despite the rather good agreement between experiment and the model description discussed so far, there are two reasons why the present situation is not yet completely satisfactory. On one hand, $\alpha=1$ corresponds to a polaron extending through the whole spin glass, on the other, it is known that, besides the percolating spin glass cluster, there exist small clusters of antiferromagnetically coupled manganese spins. It turned out that these two problems are connected, since an extended model containing bulk spin glass order and clusters of a small number of antiferrocoupled Mn spins allows to describe the experimental data with a more realistic value of $\alpha$.

We model the temperature dependent polarisation of the small Mn-spin clusters by use of the wellknfwn modified Brillouin function 17 commonly used to fit the experiments on Zeeman splitting of excitons in $(\mathrm{Cd}, \mathrm{Mn}) \mathrm{Te}$. This yields

$$
m_{c l}=x S_{\text {eff }} B_{5 / 2}\left(\frac{\frac{5}{2} \mu B_{e x c h}}{T+T_{e f f}}\right) .
$$

Here the effective spin $S_{\text {eff }}<5 / 2$ and the effective temperature $T_{\text {eff }}$ reflect the antiferromagnetic coupling of Mnspins (see Ref.17); $x=0.33$ stands for the manganese concentration, and $\mu$ denotes the Bohr magneton. In the argument of the Brillouin function we insert instead of an external magnetic field the exchange field $B_{\text {exch }}$ betwen hole spin and Mn-spins in the MP. For the manganese concentration of $33 \%$ the exchange field is $B_{\text {exch }} \approx 5.9 T 12$.

The magnetic part of the EMP energy is now given as the sum of the contribution originating in spin glass order and a second term representing the effect of small clusters; the total magnetic EMP energy then becomes

$$
E_{E M P}=\frac{N_{1}}{N_{1}+N_{c l}} \kappa p m+\frac{N_{c l}}{N_{1}+N_{c l}} \kappa_{0} p m_{c l},
$$

where $N_{c l}$ denotes the mumber of spins within small clusters in the MP. The exchange constant $\kappa_{0}=880 \mathrm{meV}$ is known from experiment 1 .

We fitted the experimental data with the form (25). Now, in addition to $\kappa, \alpha$ and $N_{1}$, we face three fit parameters more. These are the number of spins in small clusters $N_{c l}$ and the parameters of the modified Brillouin function $S_{\text {eff }}$ and $T_{\text {eff }}$. The aim of our procedure is now to prove that

i) it is possible to fit experimental data with allowance for the additional presence of small clusters, keeping all parameter values within the physically meaningful regime, together with

ii) the plateau in the temperature dependence as a genuine effect of the spin glass. This plateau cannot be obtained selfconsistently from the model of clusters of antiferromagnetically coupled spins only. The fitting procedure is illustrated by Fig. .

As mentioned above the searched for function $E_{E M P}(T)$ is determined by the temperature dependences of the magnetisations $m$ and $m_{c l}$ because the hole-spin is completely polarised in the entire temperature region under consideration. For bulk-like real systems one expects a nonzero portion of the spin glass cluster to be out of reach of the exciton wavefunction, hence outside the polaron. Therefore the real physical situation corresponds to $\alpha<1$. The ratio $N_{1} / N_{c l}$ defines the relative contribution of the SG and of small clusters to the EMP energy. The lack of experimental information on the precise value of $\alpha$ and $N_{1} / N_{c l}$ forced us to analyse fits with different values of $\alpha$ for the interval from 0.3 up to 0.7 and for $N_{1} / N_{c l}$ of order 1 . The results for the fits were remarkably insensitive to the variation of $\alpha$ at least within this regime. In the fit presented in Figs. (1) (dash-dotted line) and 5 (solid line) we fix $\alpha=0.5$ and $N_{1}=N_{c l}$, which we consider to be a good choice for the real system under consideration. While these relations were not rigorously dictated from experiment, the insensitivity of the fits with respect to variations of these two parameters over a wide range guarantees that the fit, given for the above choice, will describe the real system.

Having chosen $\alpha$ and $N_{1} / N_{c l}$, we are left effectively with four fit parameters, $\kappa, N_{1}, S_{e f f}$, and $T_{e f f}$, which remain to be determined. In the following we define the four relations employed here to determine these parameters unambiguously. The first three of these conditions are the principal experimental features, which need to be reproduced by the fit curve:

i) the absolute plateau-height at low temperatures $(\approx 28 \mathrm{meV})$,

ii) the high temperature end of the plateau ('plateau-ridge') at $\approx 3 K$, and

iii) the decay of the magnetic EMP energy at higher temperatures, as seen in the experiment way above the spin glass 
freezing.

The fourth condition is concluded from the following consideration: The plateau ridge is strongly connected with the position of the peak caused by the SG contribution (dashed line in Fig.5), which itself is determined by the value $\kappa_{1}=\kappa / N_{1}$. Keeping the position of the peak as close as possible to the plateau ridge, condition iv) results and we obtain a single value $\kappa_{1}$ for each $\kappa$; hence the parameter $N_{1}$ is now completely determined by the value of $\kappa$.

The matching of the plateau's high temperature end with the experimental data is achieved by tuning the form of the contribution of small clusters (by means of the parameters $T_{\text {eff }}$ and $S_{\text {eff }}$ ) for each $\kappa$. This requirement provides a relation between $\kappa, S_{\text {eff }}$, and $T_{\text {eff }}$.

The absolute value of the plateau is defined by the parameters $S_{\text {eff }}$ and $\kappa$, whilst the role of $T_{\text {eff }}$ and $N_{1}$ is negligible. Thus the absolute plateau height of the energy curve yields a relation between $\kappa$ and $S_{\text {eff }}$.

Finally the form of the decay at high temperatures as seen in the experiments provides one more relation between the parameters $\kappa, S_{\text {eff }}, T_{\text {eff }}$, and $N_{1}$.

Fig. 5 shows the form of the SG contribution (dashed line) and that of the small cluster contribution (dashed-dotted line) to the EMP energy together with the resulting combined curve of the temperature dependence (solid line). Note that the form of the small cluster contribution is concave. It follows that the plateau results from the SG contribution to the EMP energy.

One can try to fit the same experimental data with the modified Brillouin function alone. However, in this case one should take the parameters obtained from the Zeeman splitting fit in $\mathrm{Cd}_{0.67} \mathrm{Mn}_{0.33} \mathrm{Te}$, since now the Brillouin function describes the whole spin system and not only the part of small clusters as above. If one does so, the Brillouin fit does not coincide with the experimental data. Agreement can only be achieved by using Brillouin function fit-parameters very different from those obtained by the Zeeman splitting. For this reason such a procedure does not result in a consistent description of the complex magnetic behavior of $(\mathrm{Cd}, \mathrm{Mn}) \mathrm{Te}$.

In conclusion, the fit described above shows that the main qualitative feature, which is the plateau in the temperature dependence of the EMP energy, is the specific result of the spin glass order in the polaron. The plateau occurs at a temperature lower than the bulk SG transition temperature because of the high exchange field in the polaron.

\section{DYNAMICAL ASPECTS OF MAGNETIC POLARON FORMATION}

In this section we suggest a phenomenological model for the dynamics of the MP formation. The approach presented here aims at taking into account the contribution of the SG-phase. Thus we do not consider the nonmagnetic contributions to the MP formation (prelocalisation), the shrinking of the hole-wavefunction during MP-formation and the microscopic mechanisms of the spin- and energy relaxation (a description of these subjects is given in Ref. 25.23 .24$)$.

We suppose the changes of the averaged spin of the hole and the magnetisation in the polaron to be slow in time in comparison with the thermodynamic fluctuations so that, at each instant of time, we can define the state of the system by thermodynamically averaged quantities (magnetisation, average hole-spin etc.). Then the formation of the MP can be considered as an evolution of MP parameters $m$ and $p$ in time from zero values at $t=0$ which is the trivial solution of the static mean field equations (12 and 13) towards the nontrivial static solutions at $t \rightarrow \infty$. This evolution is governed by an additional dynamical part. Hence we propose the following phenomenological set of equations

$$
\begin{gathered}
\ddot{p}+\gamma \dot{p}+\Omega^{2} p=\Omega^{2} \tanh [\beta \kappa m], \\
\ddot{m}+\gamma \dot{m}+\Omega^{2} m=\Omega^{2} \int_{G} d z \tanh \left[\beta\left(J \sqrt{q} z+\kappa \frac{p}{N_{1}}\right)\right] .
\end{gathered}
$$

This form of the dynamical part contains only the leading time derivatives and is linear in $p$ and $m$. An alternative form containing only first time derivatives does not yield a time evolution under the starting conditions $p=0, m=0$. The Eqs. (26), (27) describe the dynamics of nonlinearly coupled harmonic oscillators with damping. The characteristic frequency $\Omega$ is the oscillation frequency, while $\gamma$ characterises the damping.

We assume $q$ to satisfy equation (14) with time-dependent $p$ and $m$. This assumption is consistent with the definition of $q$ as Edwards-Anderson order parameter. The equilibration time for $q$ is of order $1 / T$ - the characteristic time of thermodynamic relaxation processes at this temperature. For the suggested model this time is shorter than the characteristic evolution time of $p$ and $m$.

The dynamics of the MP formation is described by the solution of equations (26), (27), (14) with the initial conditions $p(t=0)=0, m(t=0)=0$. We can obtain an analytical solution for the initial stage of the MP formation where 
$\beta \kappa m \ll 1, \beta \kappa p / N_{1} \ll 1$ so that we may expand in these values up to the linear terms. In the paramagnetic phase $q$ is nonzero only due to the exchange field of the MP. The relevant values of $z$ in the integral of Eq. (27) lie in the region $J \sqrt{q} z \ll \kappa p / N_{1}$. Hence we neglect $q$ in Eq.(27) and obtain for the initial step of MP-formation $t \ll \min (1 / \gamma, 1 / \Omega)$ in the paramagnetic phase $(T>J)$

$$
\begin{gathered}
\ddot{p}+\gamma \dot{p}+\Omega^{2} p=\Omega^{2} \beta \kappa m, \\
\ddot{m}+\gamma \dot{m}+\Omega^{2} m=\Omega^{2} \beta \kappa \frac{p}{N_{1}} .
\end{gathered}
$$

The nontrivial solution of this system of linear differential equations with the initial conditions $m(0)=0, p(0)=0$ is

$$
\begin{gathered}
m=C e^{-\gamma t / 2} \sinh \left[\frac{t}{2} \sqrt{\gamma^{2}+4 \Omega^{2}\left(\beta \kappa / \sqrt{N_{1}}-1\right)}\right], \\
p=C \sqrt{N_{1}} e^{-\gamma t / 2} \sinh \left[\frac{t}{2} \sqrt{\gamma^{2}+4 \Omega^{2}\left(\beta \kappa / \sqrt{N_{1}}-1\right)}\right] .
\end{gathered}
$$

One obtains a nonoscillating solution, which corresponds to the formation of the polaron only under the condition $\beta \kappa / \sqrt{N_{1}}-1>0$. The same inequality was obtained in the end of section II from the static selfconsistency equations as a necessary condition for the polaron formation. The constant $C$ and the characteristic frequencies $\gamma$ and $\Omega$ could be inferred from the comparison with experiment.

Let us now consider the case of spin glass order. In the vicinity of the phase transition the integral in Eq. (27) is dominated by $\beta J \sqrt{q} z \ll 1$. Since $\beta \kappa p / N_{1} \ll 1$, we have

$$
\begin{aligned}
\int_{G} d z \tanh \left[\beta\left(J \sqrt{q} z+\kappa \frac{p}{N_{1}}\right)\right] & \approx \tanh \left(\beta \kappa p / N_{1}\right)\left(1-\int_{G} d z \tanh ^{2}(\beta J \sqrt{q} z)\right) \\
& \equiv \tanh \left(\beta \kappa p / N_{1}\right)(1-I(q))
\end{aligned}
$$

At times $t \ll 1 / \gamma$ the condition $J \sqrt{q} z \gg \kappa p / N_{1}$ is fulfilled for the relevant domain of $z$ in the integral. Then we can set $I(q) \approx q$ with $q$ evaluated from Eq.(14) with $p=0$. Expanding $\tanh (x) \approx x$ the following equations are obtained

$$
\begin{gathered}
\ddot{p}+\gamma \dot{p}+\Omega^{2} p=\Omega^{2} \beta \kappa m, \\
\ddot{m}+\gamma \dot{m}+\Omega^{2} m=\Omega^{2} \beta \kappa \frac{p}{N_{1}} G(q),
\end{gathered}
$$

where $G(q)=1-I(q) \approx 1-q$. The solutions of these equations read

$$
\begin{gathered}
m=C e^{-\gamma t / 2} \sinh \left[\frac{t}{2} \sqrt{\gamma^{2}+4 \Omega^{2}\left(\beta \kappa \sqrt{G(q) / N_{1}}-1\right)}\right], \\
p=C \sqrt{N_{1} / G(q)} e^{-\gamma t / 2} \sinh \left[\frac{t}{2} \sqrt{\gamma^{2}+4 \Omega^{2}\left(\beta \kappa \sqrt{G(q) / N_{1}}-1\right)}\right] .
\end{gathered}
$$

¿From $G=1-q<1$ it follows that the spin glass order slows down the magnetic polaron formation.

The time dependence of the exchange part of the MP energy at the initial stage of MP formation (Eq. (23)) is given by

$$
\epsilon(t) \propto \sqrt{N_{1} / G(q)} e^{-\gamma t} \sinh ^{2}\left[\frac{t}{2} \sqrt{\gamma^{2}+4 \Omega^{2}\left(\beta \kappa \sqrt{G(q) / N_{1}}-1\right)}\right]
$$

where 


$$
G(q) \equiv \begin{cases}1 & \text { in paramagnetic phase } \\ 1-I(q) & \text { in spin-glass phase. }\end{cases}
$$

Thus the MP formation depends exponentially on time. Representing the magnetic part of the MP energy as $\epsilon(t) \propto$ $\exp \left(t / \tau_{f}\right)$, we obtain the following temperature dependence for the MP formation time

$$
\tau_{f} \equiv\left(\sqrt{\gamma^{2}+4 \Omega^{2}\left(\beta \kappa \sqrt{G(q) / N_{1}}-1\right)}-\gamma\right)^{-1}
$$

The introduced model allows one to discriminate two limiting cases of the MP formation dynamics: the case of "strong dissipation" $\gamma \gg \Omega$ and the case of "weak dissipation" $\Omega \gg \gamma$. The expressions for the MP formation time in these two limit cases read

$$
\tau_{f} \approx \begin{cases}{\left[\frac{\Omega^{2}}{\gamma}\left(\beta \kappa \sqrt{G(q) / N_{1}}-1\right)\right]^{-1},} & \text { for } \gamma \gg \Omega, \\ {\left[2 \Omega\left(\beta \kappa \sqrt{G(q) / N_{1}}-1\right)^{1 / 2}\right]^{-1},} & \text { for } \Omega \gg \gamma\end{cases}
$$

Neglecting the contribution of the exchange field to $q$ in the SG phase $(q \propto(J-T) / J)$ one can see the crossover of the temperature dependence of the MP formation time at the SG transition. At $\gamma \gg \Omega$ the crossover is from $\tau_{f} \propto T$ in the paramagnetic phase to $\tau_{f} \propto T^{1 / 2}$ in the SG phase. At $\Omega \gg \gamma$ the crossover is from $\tau_{f} \propto T^{1 / 2}$ in the paramagnetic phase to $\tau_{f} \propto T^{1 / 4}$ in the SG-phase. The qualitatively different temperature behaviors of the MP formation time in the different extreme cases give a possibility to infer from the comparison with experiment the relevant corresponding dynamical model. The limit case of "strong dissipation" coincides with the dynamical model introduced in the paper $15,23.25$.

Taking a typical value of the exciton magnetic polaron formation time of order $100 \mathrm{ps}$ at temperature $T \approx 2 \mathrm{~K} 1 \mathrm{~L}, 23$ and the parameters $\kappa=41 \mathrm{meV}, N_{1}=20$ (the parameters of the solid line in Fig.(4), we can evaluate the order of magnitude of the characteristic frequences $\Omega$ and $\gamma$. For the "weak dissipation" case $(\Omega \gg \gamma)$ we find $\Omega \sim 10^{9} \mathrm{~Hz}$. For the case of "strong dissipation" $\left(\gamma \gg \Omega\right.$ ) we find $\Omega^{2} / \gamma \sim 10^{8} \mathrm{~Hz}$, which implies $\gamma \gg \Omega \gg 10^{8} \mathrm{~Hz}$. The evaluation with the parameters $\kappa=65 \mathrm{meV}$ and $N_{1}=50$ corresponding to the dashed-dotted line in Fig. 1 would give the same order of the characteristic frequencies.

\section{CONCLUSION}

We elaborated a detailed model description of specific thermodynamic features of the magnetic polaron near the spin glass (SG) transition and due to SG order. The effect of the exchange interaction between carrier-spin and the spin glass is analogous to the effect of a spatially confined exchange magnetic field, which competes with SG order. Consequently, this field leads to both a decreased and smeared freezing temperature within the range of the magnetic polaron. The onset of spin glass order results in a qualitative change of the temperature dependence of the magnetic polaron energy as observed experimentally. The obtained solutions of the selfconsistent equations for the polaron magnetisation and for the thermodynamically averaged hole spin allowed to fit the experimental data of the temperature dependence of the exciton magnetic polaron energy in $(\mathrm{Cd}, \mathrm{Mn}) \mathrm{Te}$.

An extended model describing different dynamical scenarios of the magnetic polaron formation was introduced. These dynamical classes can be distinguished by specific thermal behavior of the MP formation time. A comparison with experiment on thermal behaviour should hence allow to identify the relevant dynamical scenario.

\section{ACKNOWLEDGMENTS}

We wish to acknowledge helpful discussions with Dr. Cl. Rigaux, Dr. A. Mauger, and collaborators at Universite Paris VI et VII, and with Dr.M. Dahl at Würzburg university.

This work was supported by the Deutsche Forschungsgemeinschaft through the Sonderforschungsbereich 410. 
* on leave from A.F. Ioffe Physico-Technical Institute, Russian Academy of Sciences, 194021 St. Petersburg, Russia.

${ }^{1}$ J.K. Furdyna, J. Appl. Phys. 64, R29 (1988)

${ }^{2}$ G. Mackh, W. Ossau, D.R. Yakovlev, A. Waag, T. Litz, and G. Landwehr, Solid State Commun. 88, 221 (1993)

${ }^{3}$ A. Mauger, N.S. Almeida and D.L. Mills, Phys. Rev. B38, 1296 (1988)

${ }^{4}$ C.E.T. Gonsales da Silva, Phys. Rev. B 33, 2923 (1986)

${ }^{5}$ G. Mackh, W. Ossau, D.R. Yakovlev, G. Landwehr, T. Wojtowicz, G. Karczewski, and J. Kossut, Acta Physica Polonica A88, 849 (1995)

${ }^{6}$ P.A. Wolff, in Semiconductors and Semimetals Vol.25, ed. by J.K. Furdyna and J. Kossut, p.413 (Academic Press, London, 1988)

7 J. Villain, Z. Physik B33, 31 (1979)

${ }^{8}$ A. Mauger, J. Villain, Y. Zhou, C. Rigaux, N. Bontemps, and J. Ferre, Phys. Rev. B41, 4587 (1990)

${ }^{9}$ J. Stein, private communication and Europhys. Lett. 34, 717 (1996)

${ }^{10}$ S. Oseroff and P.H. Keesom, in Semiconductors and Semimetals 25, 73 (1988)

11 A.V. Kudinov, Yu.G. Kusraev, and V.N. Yakimovich, Phys. Solid State 37, 359 (1995)

12 I.A. Merkulov, D.R. Yakovlev, K.V. Kavokin. G. Mackh, W. Ossau, A. Waag, and G. Landwehr JETP Lett. 62, 335 (1995)

${ }^{13}$ D.R. Yakovlev, U. Zehnder, W. Ossau, A. Waag, G. Landwehr, T. Wojtowicz, G. Karczewski, and J. Kossut, to be published

${ }^{14}$ G. Mackh, W. Ossau, D.R. Yakovlev, A. Waag, G. Landwehr, R. Hellmann, and E.O. Göbel, Phys. Rev. B49, 10248 (1994)

15 T. Dietl, P. Peyla, W. Grieshaber, and Y. Merle d'Aubigne, Phys. Rev. Lett. 74, 474 (1995)

${ }^{16}$ Diluted Magnetic Semiconductors, Ed. by J.K. Furdyna, J. Kossut (Academic Press, London, 1988)

17 J.A. Gaj, R. Planel, and G. Fishman, Solid State Commun. 29, 435 (1979)

${ }^{18}$ K. Binder and A.P. Young, Rev. Mod. Phys. 58, 801 (1986)

${ }^{19}$ K.H. Fisher and J.A. Hertz, Spin Glasses, Cambridge University Press, 1991

${ }^{20}$ D. Sherrington and S. Kirkpatrick, Phys. Rev. Lett. 35, 1792 (1975)

${ }^{21}$ S.M. Ryabchenko and Yu.G. Semenov, Sov. Phys. JETP 57, 825 (1983); I.A. Merkulov and K.V. Kavokin, Phys. Rev. B52, 1751 (1995)

${ }^{22}$ A.V. Kavokin and K.V. Kavokin, Semicond. Sci. Technol. 8, 191 (1993); C. Benouit a la Guillaume, Phys. Stat. Sol. (b) 175, 369 (1993)

${ }^{23}$ D.R. Yakovlev and K.V. Kavokin, Comments Cond. Mat. Phys. 18, 51 (1996)

${ }^{24}$ K.V. Kavokin, Sov. Phys. Solid State 35, 818 (1993)

25 The coincidence between the "strong dissipation" scenarip and the dynamics described in the present paper 23 is obtained if one identifies $\Omega^{2} / \gamma$ in our model with $1 / \tau_{S}$ in Eq.(1) of 23 . 


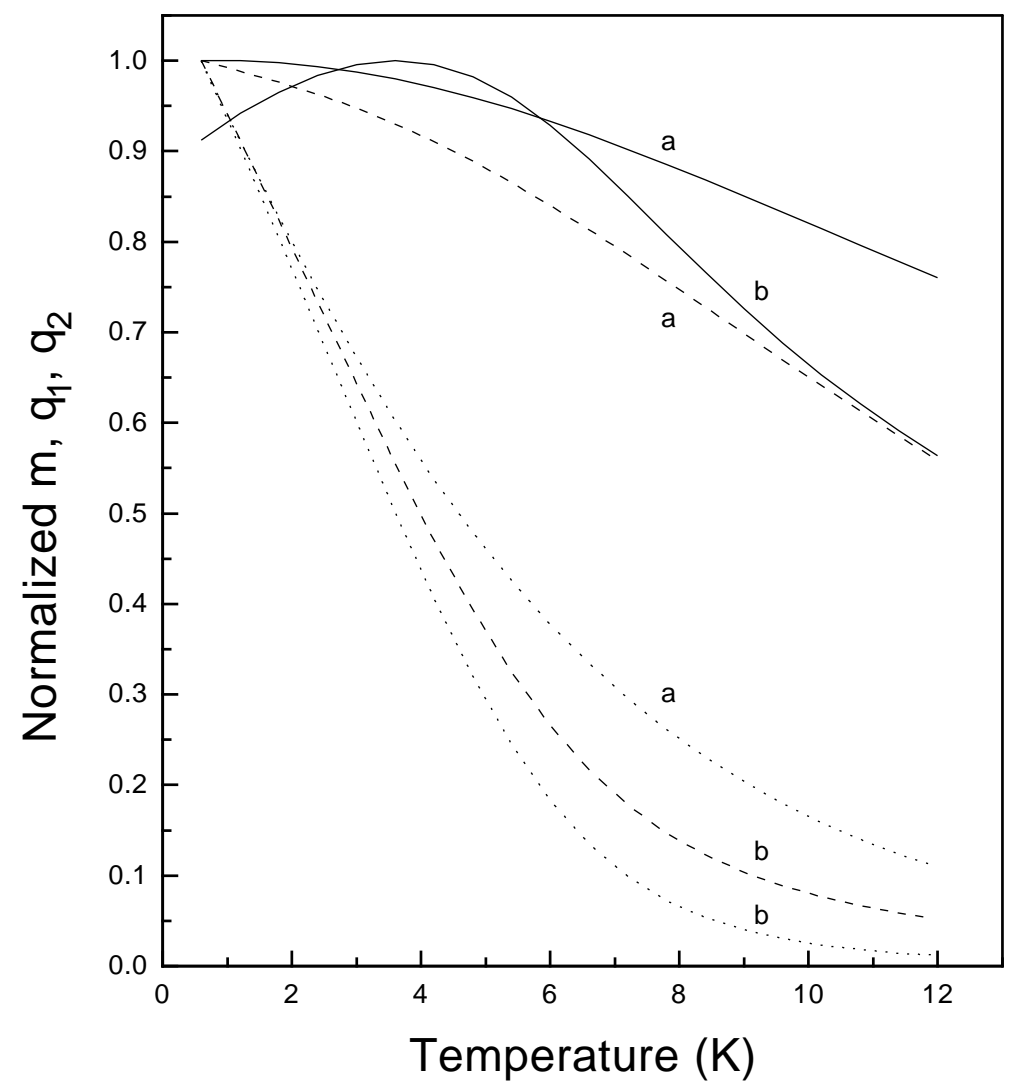

Figure 1

FIG. 1. Temperature dependences of the magnetization $m$ (solid lines) and SG order parameters $q_{1}$ (dashed lines) and $q_{2}$ (dotted lines) at two different values of exchange constant $\kappa_{1}=\kappa / N_{1}$ : (a) $\kappa_{1}=3.14 \mathrm{meV}$ and (b) $\kappa_{1}=0.628 \mathrm{meV}$. Parameters $\kappa=44 \mathrm{meV}$ and $\alpha=0.7$ are fixed. The curves are normalized to the largest value of the corresponding parameter. 


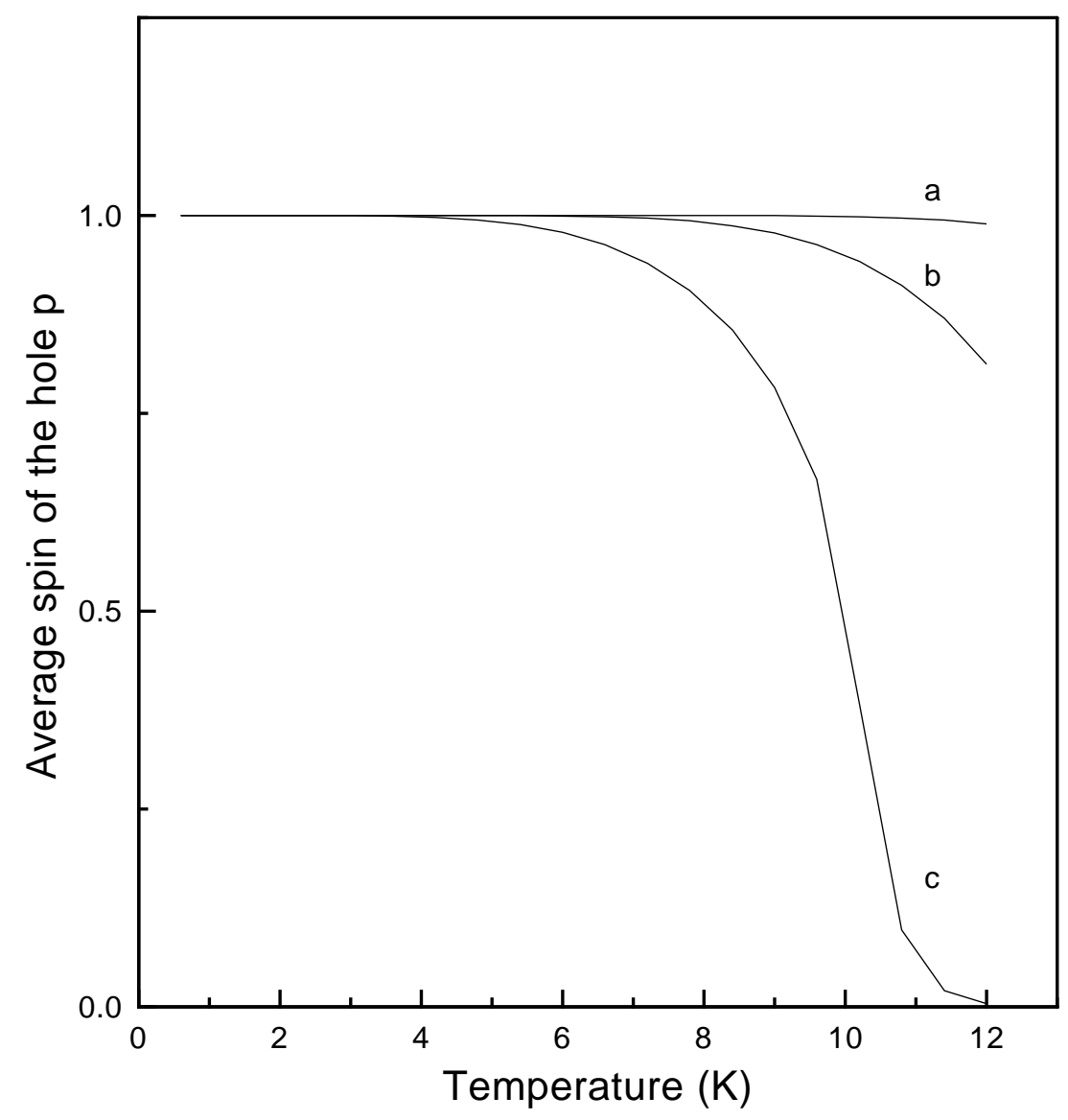

Figure 2

FIG. 2. The temperature dependence of the average spin of the hole $p(T)$ for different $\kappa$ : (a) $\kappa=10 \mathrm{meV}$, (b) $\kappa=5 \mathrm{meV}$, (c) $\kappa=3 \mathrm{meV}$. The parameters $J=8 K, \alpha=0.7, h=0$, and $\kappa_{1}=0.63 \mathrm{meV}$ are fixed. 


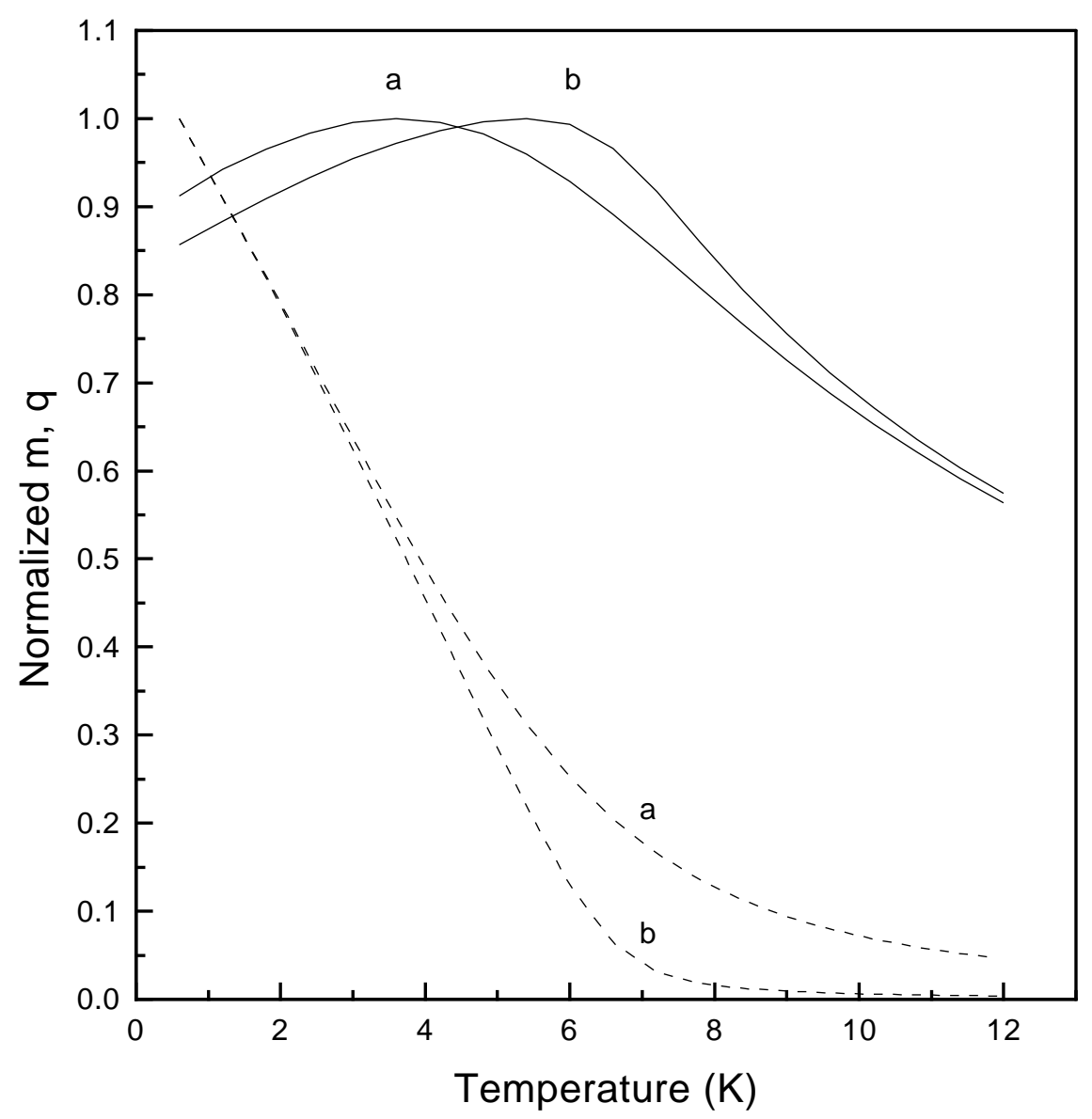

Figure 3

FIG. 3. Temperature dependences of the magnetization $m$ (solid lines) and the SG order parameter $q=\alpha q_{1}+(1-\alpha) q_{2}$ (dashed lines) at two different values of $\alpha$ : (a) $\alpha=0.7$ and (b) $\alpha=0.2$. Other parameters are fixed: $J=8 K, \kappa_{1}=0.628 m e V$, $\kappa=44 \mathrm{meV}$. 


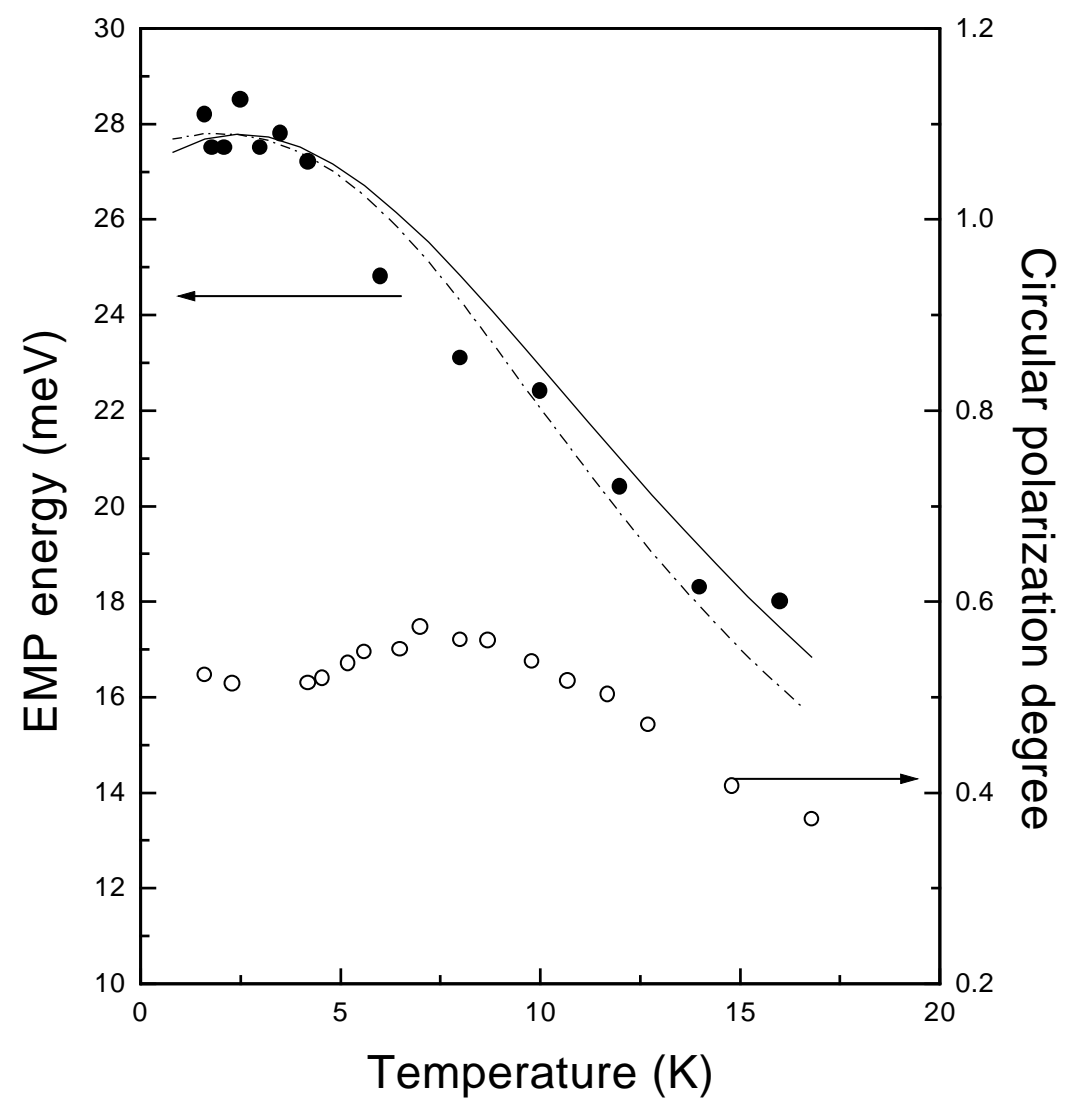

Figure 4

FIG. 4. The temperature dependence of EMP energy (closed circles) and the magnetic-field-induced polarization degree measured at $0.4 \mathrm{~T}$ (open circles) of a MBE-grown epilayer $C d_{0.67} M n_{0.33} T e$. Results of theoretical calculations: Solid line: Fit with SG only, $\kappa=41 \mathrm{meV}, N=20, \alpha=1$. Dashed-dot line: Fit with SG and small clusters, $\kappa_{0}=880 \mathrm{meV}, T_{e f f}=8 K$, $S_{\text {eff }}=0.12, \kappa=65 \mathrm{meV}, N=100, \alpha=0.5, N_{1}=N_{c l}=50$. 


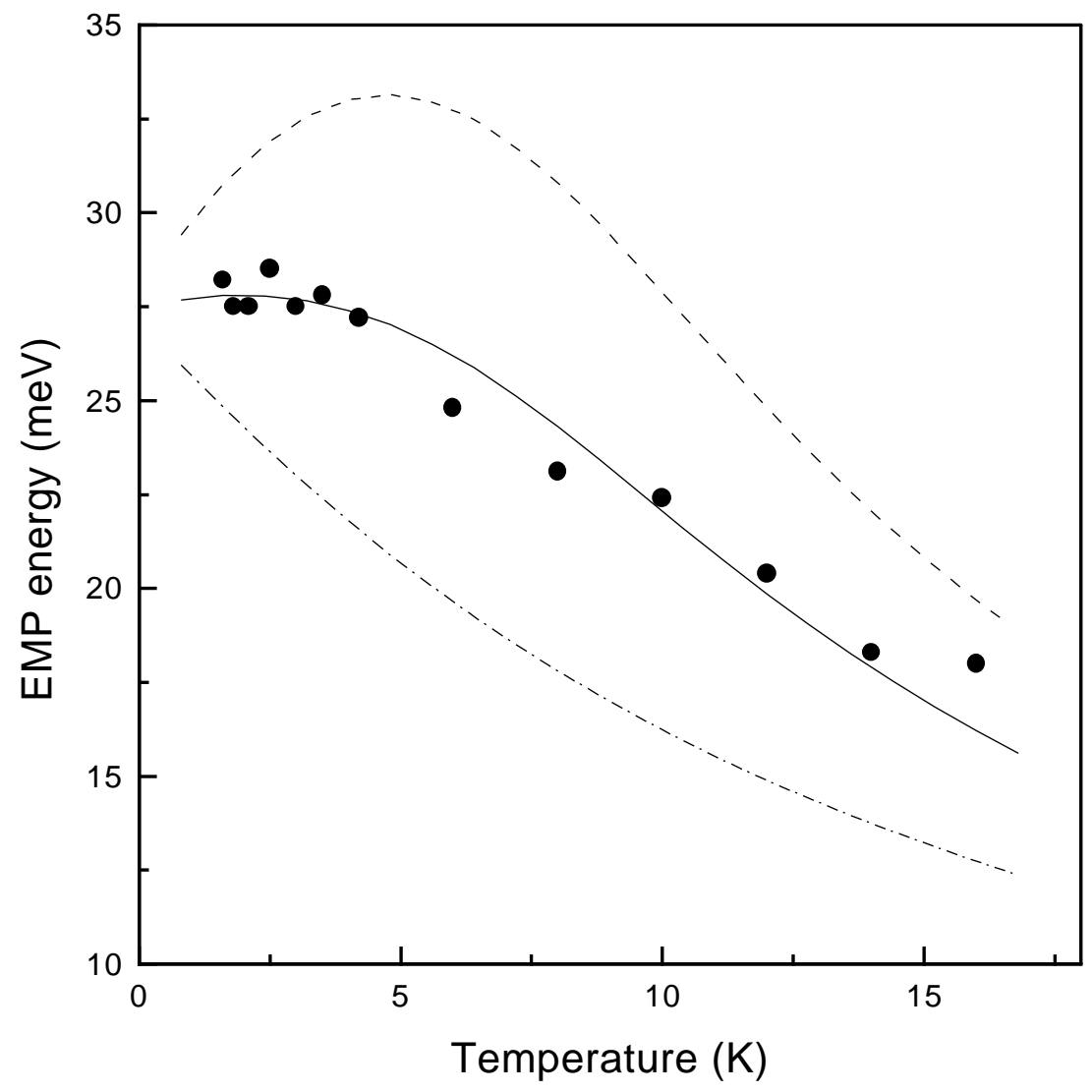

Figure 5

FIG. 5. The fitting procedure for the model of SG and small spin clusters. Solid circles: experimental points as in Fig. 1 . Dashed line: the SG contribution to the EMP energy. Dashed-dotted line: the contribution of small clusters to the EMP energy. Solid line: the resulting fit curve. The parameters of dashed, dashed-dotted and solid lines are the same as by the dashed-dotted line in Fig. 4 